15 | 1993

Varia

\title{
Pascal au pays du matin calme
}

\section{Ran-E Hong}

\section{OpenEdition}

Journals

Édition électronique

URL : http://journals.openedition.org/ccibp/596

DOI : $10.4000 /$ ccibp. 596

ISSN : 2493-7460

\section{Éditeur}

Centre international Blaise Pascal

\section{Édition imprimée}

Date de publication : 27 février 1993

Pagination : 6-7

ISSN : 0249-6674

\section{Référence électronique}

Ran-E Hong, "Pascal au pays du matin calme », Courrier du Centre international Blaise Pascal [En ligne], 15 | 1993, mis en ligne le 07 janvier 2016, consulté le 14 septembre 2020. URL : http:// journals.openedition.org/ccibp/596

Ce document a été généré automatiquement le 14 septembre 2020.

Centre international Blaise Pascal 


\title{
Pascal au pays du matin calme
}

\author{
Ran-E Hong
}

1 Après le Japon, la Corée à son tour jouit d'une certaine florescence dans les études pascaliennes. Celles-ci sont largement dues à la qualité des travaux et de l'enseignement de celui qui en reste le doyen, à savoir $\mathrm{M}$. Lee Whan ${ }^{1}$. Le premier à avoir, en 1974, consacré une thèse de doctorat sur Pascal, il s'intéresse tout particulièrement à la première partie de l'Apologie communément appelée l'anthropologie de Pascal. Dans son livre majeur Recherches sur Pascal qui constitue un recueil d'articles sur Pascal, il dépeint la situation de l'homme sans Dieu et analyse les diverses sectes philosophiques tout en faisant ressortir la critique que Pascal émet à l'égard de celles-ci. Mais l'anthropologie n'a de valeur que parce qu'elle mène à la théologie, point sur lequel la réflexion de $\mathrm{M}$. Lee s'attarde tout particulièrement. M. Lee est l'auteur également d'une traduction qui est aussi une édition critique des Pensées (Pascal. Les Pensées) basée sur l'édition Lafuma (Paris, Delmas, 1960) qui demeure la plus fidèle des traductions coréennes, quoiqu'elle ne soit malheureusement pas intégrale.

2 Puis vient une génération de jeunes pascalisants qui, pour la plupart, sont des élèves de M. Lee et ont fait leurs études en France.

$3 \mathrm{M}^{\mathrm{lle}}$ Cho, déjà connu par sa thèse sur Pascal et Lao-Tseu, poursuit ses études comparatives dans un article aussi dense que pertinent paru dans le livre du colloque sur Pascal. Port-Royal. Orient. Occident.

$4 \quad \mathrm{M}^{\text {lle }} \mathrm{Bai}$, de son côté, consacre sa thèse à l'idée de coutume dans les Pensées de Pascal. Dans un premier temps, elle esquisse l'influence de Montaigne sur la notion de coutume mise en rapport avec celle de nature. Puis, par une lecture attentive des fragments du pari et grâce à l'éclairage procuré par l'analyse de la machine cartésienne, elle cerne le rôle de la coutume dans l'apologétique pascalienne et montre bien que la coutume assume « une fonction médiatrice » entre la raison et l'inspiration. $\mathrm{M}^{\text {lle }}$ Bai ne manque pas, à juste droit, de traiter du point de vue juridique de la notion de coutume, contenu par l'exemple dans l'expression « droit coutumier ».

5 L'étude de M. Hyung-Kil Kim² De l'art de persuader dans les Pensées de Pascal constitue un extrait de sa thèse du même titre, soutenue à l'université de Provence en 1989. Dans ce travail dans lequel il faut saluer la première thèse coréenne sur Pascal publiée en 
France, l'auteur s'attache à montrer que la théorie pascalienne de la persuasion telle qu'elle apparaît dans l'opuscule De l'art de persuader a été mise en pratique dans les Pensées. Pour ce faire, il relève méthodiquement tous les termes primitifs et toutes les propositions appartenant à la logique et constate que l'ordre de l'Apologie relève autant de l'ordre géométrique, rigoureux que de l'ordre plus souple qu'est celui de la digression.

6 Enfin, dans sa thèse, intitulé Arrière-plan de la pensée de Pascal M. Bo-Kyun Kim se fait fort non pas tant de faire un examen précis des sources des Pensées mais plutôt d'offrir une vaste fresque des diverses influences exercées sur la pensée de Pascal. Avec une large érudition, l'auteur s'attache à relever en particulier les ressemblances entre la pensée de Pascal et celle des premiers Pères de l'Église, saint Paul, Tertullien puis avec celle de saint Augustin et de Saint Cyran. Puis s'appuyant largement sur L'entretien de Pascal avec M. de Sacy, il relève les parentés entre Pascal et Montaigne, puis entre Pascal et Épictète tout en insistant sur les points sur lesquels ces deux auteurs sont combattus par Pascal.

7 Les recherches de ces différents chercheurs sont stimulées par des rencontres, conférences (Jean Mesnard en 1988) et communications ( ${ }^{\text {lle }} \mathrm{Bai}^{3}$ et $\mathrm{M}$. Hyung-Kil Kim ${ }^{4}$ en 1989) tenues ici et là. On regrettera toutefois que ces recherches restent quelque peu concentrées sur la seule œuvre des Pensées et sur le seul aspect de Pascal penseur.

8 Mais malgré ses débuts prometteurs, ne sommes-nous pas ici encore le matin, un matin calme? Souhaitons que dans un proche avenir d'autres vocations se fassent jour et puissent montrer toute la mesure de leur talent.

BIBLIOGRAPHIE

\section{Thèses de doctorat en soutenues par des Coréens en France et en Corée}

LEE, Whan, L'anthropologie de Pascal dans les Pensées, Université de Séoul, 1974. (en coréen).

HONG, Ran-E, Le dialogue chez Pascal. De la forme dialoguée à la dialectique, Université de de Paris IV, 1986, (voir CIBP nº et 9).

CHO, Myung-Ai, L'homme et Dieu chez Pascal et Lao-Tseu, Université de Paris IV, 1987, (voir CIBP $\left.n^{\circ} 9\right)$.

BAI, Seong-Ok, L'idée de coutume dans les Pensées de Pascal (aspect moral, théologique et apologétique de la notion), Université de Paris IV, 1988.

KIM, Hyung-Kil, De l'art de persuader dans les Pensées de Pascal, Université de Provence, 1989 (voir CIBP $\left.n^{\circ} 11\right)$.

KIM, Bo-Kyun, Arrière-plan de la pensée de Pascal, Université Hankook des Études Étrangères, 1990 (en coréen). 


\section{Ouvrages publiées par des chercheurs coréens}

LEE, Whan, Recherches sur Pascal, Séoul, Minoumsa, 1974 (en coréen).

LEE, Whan, La vie et la pensée de Pascal, Séoul, Pakyoungsa, 1976 (en coréen).

LEE, Whan, Pascal. Les Pensées, Séoul, Presses de l'Université de Séoul, 1985 (en coréen).

KIM, Hyung-Kil, De l'art de persuader dans les Pensées de Pascal, Paris, Nizet, 1992.

\section{Articles publiés par des chercheurs coréens}

KIM, Hyung-Kil, « La méthode divine dans le « Sur la conversion du pécheur » », Études de langue et de littérature françaises, 1981, n 16, pp. 123-34.

KIM, Hyung-Kil, « La méthode scientifique dans les Pensées de Pascal », Études de langue et de littérature françaises, 1983, n 18, pp. 115-31. (en coréen).

KIM, Hyung-Kil, « La figure de l'ironie dans les Pensées de Pascal », Cahiers de langue et de littérature françaises de l'Université Jeonju, $\mathrm{n}^{\circ}$ 1, pp. 5-23.

KIM, Hyung-Kil, « La vision augustinienne du monde dans les Pensées de Pascal », Études de langue et de littérature françaises, 1986, $\mathrm{n}^{\circ} 21, \mathrm{pp} .171-206$.

KIM, Hyung-Kil, « Le concept d'ordre dans les Pensées de Pascal », Études de langue et de littérature françaises, $1990, \mathrm{n}^{\circ} 25$, pp. 67-77.

CHO, Myung-Ai, « Pascal et Lao-Tseu : l'union des contraires », Pascal. Port-Royal. Orient. Occident, Paris, Klincksieck, 1991, pp. 89-98.

HONG, Ran-E, « Le paradoxe dans les premières liasses de l'Apologie pascalienne », Cahiers de l'Association Internationale des Études Françaises, 1988, n 40, pp. 273-83.

HONG, Ran-E, « La force et ses aspects chez Pascal », Équinoxe, 1988, n³, pp. 19-26.

HONG, Ran-E, « Petite étude sur Pascal (1) », Mélanges offerts à Chung Byung-Hee pour son $60^{e}$ anniversaire, Séoul, Samwha, 1989, pp. 273-87 (en coréen).

HONG, Ran-E, « L'honnête homme selon Pascal et la figure de l'homme de qualité selon Confucius ", Pascal. Port-Royal. Orient, Occident., Paris, Klincksieck, 1991, pp. 63-69.

HONG, Ran-E, « Petite études sur Pascal (2) », Études de langue et de littérature françaises, 1991, n²6, pp. 397-409 (en coréen).

HONG, Ran-E, « La dyade et la triade dans l'écriture pascalienne », Études de langue et de littérature françaises, 1993, n² 28 , pp. 343-354.

\section{NOTES}

1. Lee Whan, Université nationale de Séoul.

2. Hyung-Kil Kim, Université Jeonju.

3. Seong-Ok Bai, Université de Séoul.

4. Bo-Kyun Kim, Universités Kyushu Sankyo et Fukuoka (Japon). 
INDEX

Mots-clés : Pascal, études

Keywords : Pascal, studies

Index géographique : Corée

\section{AUTEUR}

RAN-E HONG

Université coréenne des études étrangères, Séoul 